\title{
Multicriteria synthesis of flexible heat-exchanger networks with uncertain source-stream temperatures
}

\author{
Cheng-Liang Chen*, Ping-Sung Hung \\ Department of Chemical Engineering, National Taiwan University, Taipei 10617, Taiwan, ROC
}

Received 23 May 2003; received in revised form 28 July 2003; accepted 31 March 2004

Available online 2 July 2004

\begin{abstract}
A multi-criteria synthesis strategy for heat-exchanger networks (HENs) simultaneously considering minimum utility consumption, maximum source-stream temperature flexibility, and even minimum number of matches is proposed. The flexible HEN synthesis problem is formulated as a multi-objective mixed-integer linear programming (MO-MILP). For handling the multiple conflict design targets, a two-phase fuzzy multi-criteria decision-making method is presented to attain a best compromised solution. Two numerical examples with flexibility preferences in source-stream temperatures are supplied, demonstrating that the proposed strategy can provide definite and feasible compensatory solutions for multi-criteria HEN synthesis problems.
\end{abstract}

(C) 2004 Elsevier B.V. All rights reserved.

Keywords: Heat-exchanger network; Synthesis; Flexibility; Superstructure; MILP; Multi-criteria decision-making; Fuzzy optimization

\section{Introduction}

A heat exchanger network (HEN) synthesis problem can be described as the one that synthesize a HEN configuration to reach some assigned targets such as minimum utility consumption, minimum total number of heat exchangers, etc., with given heating/cooling utilities and hot/cold process streams be cooled/heated from nominal inlet temperatures to specified target temperatures [1].

Most of the existing HEN synthesis methods rely on either heuristic rules (for example, pinch analysis method [2]) or mathematical programming (for example, simultaneous optimization approach [3-6]). And further, to some typical objectives considered in the HEN synthesis such as utility consumption, total number of matches, and total exchanger area, the flexibility of the HENs for feasible operation under possible variation of source-stream temperatures and/or heat-capacity flow rates has been emphasized in some recent articles [6-10]. For HEN synthesis, the analysis of this flexibility, defined as the size of the region of feasible operation in the space of desired or undesired deviations of pa-

\footnotetext{
* Corresponding author. Tel.: +886 2 23636194; fax: +886223623040 .

E-mail address: ccl@ntu.edu.tw (C.-L. Chen).
}

rameters from their nominal values [10], however, attracts attention usually indirectly as test examples basing on mathematical programming synthesis. Therein, [10] explored the HEN synthesis problem with simultaneous flexibility targeting and minimum-utility objective based on an MILP formulation. The optimal solution is examined on the basis of the vertices of the polyhedral uncertainty region in the space of source-stream temperatures. It is found, however, that the resulting HEN structures with increasing flexibility requirements are prone to variation, and the true maximum flexibilities of resulting HEN structures are usually greater than the assigned targets. Thus, for a given flexibility target, it is very often to obtain a more conservative HEN design by the method of simultaneous flexibility targeting and synthesis of minimum-utility HENs proposed in [10].

In this paper, we extend the work of [10] by simultaneously considering minimization of the total utility consumption, maximization of operational flexibility to source-stream temperatures, and even minimum number of matches as multiple design objectives. The flexible HEN synthesis problem is thus formulated as the one of multi-objective mixed-integer linear programming (MO-MILP). This formulation also assumes that the feasible region in the space of uncertain input parameters is convex, so that the optimal solution can thus be explored on the basis of the vertices 
of the polyhedral region of uncertainty [10]. Under the assumption of convexity, only the source-stream temperatures of the HENs are considered to be the uncertain input parameters. With this formulation, the standard definition of the HEN synthesis problem with minimal total utility consumption and even minimum number of units is extended to include a flexibility specification for the potential HEN structure and can be stated as: "given hot/cold streams to be cooled/heated from nominal supply temperatures to specified target temperatures and hot/cold utility specifications, synthesize a HEN such that it has minimal utility consumption (considering nominal case or average of all vertical operating points), minimal number of matches if desired, and maximal flexibility for feasible operation."

For handling the multiple and conflict design objectives, a fuzzy decision-making method is adopted to attain the compromised solution among all conflict objectives [11]. Therein each design objective is treated as a fuzzy goal, and a specific membership function is used to characterize the transition from the numerical objective value to the degree of satisfaction for the fuzzy objective. The final decision, therefore, is interpreted as a fuzzy aggregation of these multiple objectives and measured by the overall degree of satisfaction. And the best compromised solution is finally reached by maximizing the overall degree of satisfaction for the decision. In the course of finding the solution, two popular operators, the minimum and the average, are applied as the fuzzy intersection operators, the effects of which are examined as well. We also proposed an interactive two-phase fuzzy decision-making method by combining these two operators to take advantages of the both $[11,12]$. The minimum operator is used in phase I to maximize the degree of satisfaction for the worst objective, and the average operator is then applied in phase II to simultaneously promote satisfaction levels of all objectives with guaranteed least satisfaction value.

Two numerical examples with flexibility preference in source-stream temperatures is presented here to demonstrate that the proposed interactive two-phase fuzzy optimization method can provide a feasible and better compensatory solution for multi-objective HEN synthesis.

\section{Model formulation}

Consider the standard HEN synthesis problem with $N_{\mathrm{H}}$ hot and $N_{\mathrm{C}}$ cold process streams along with hot and cold utilities. Since it is suitable for formulating the simultaneous solution which involving the consideration of both utility consumption and operational flexibility, the HEN superstructure proposed by $[3,4]$ is applied for modeling the structure. Therein, the isothermal mixing assumption in the simplified superstructure eliminates the need for nonlinear/nonconvex energy balance. The minimum number of superstructure stages, $N_{T}$, corresponds to $\max \left\{N_{\mathrm{H}}, N_{\mathrm{C}}\right\}$, as suggested by [3]. Fig. 1 illustrates a 2-hot/2-cold/2-stage superstructure. The mathematical programming formulation for minimizing utility consumption with specified uncertain source-stream temperature ranges can be summarized as follows: $[3,4,10]$ :

$$
\begin{aligned}
& \min _{\mathbf{x} \in \Omega} J^{(0)}=\sum_{i \in \mathrm{HP}} q c u_{i}^{(0)}+\sum_{j \in \mathrm{CP}} q h u_{j}^{(0)} \\
& \boldsymbol{x} \equiv\left\{\begin{array}{r}
z_{i j k}, z c u_{i}, z h u_{j} ; t_{i k}, t_{j k} ; \mathrm{d} t_{i j k}, \mathrm{~d} t c u_{i}, \mathrm{~d} t h u_{j} ; \\
q_{i j k}, q c u_{i}, q h u_{j} ; \\
i \in \mathrm{HP}, j \in \mathrm{CP}, k \in \mathrm{ST}
\end{array}\right\}
\end{aligned}
$$

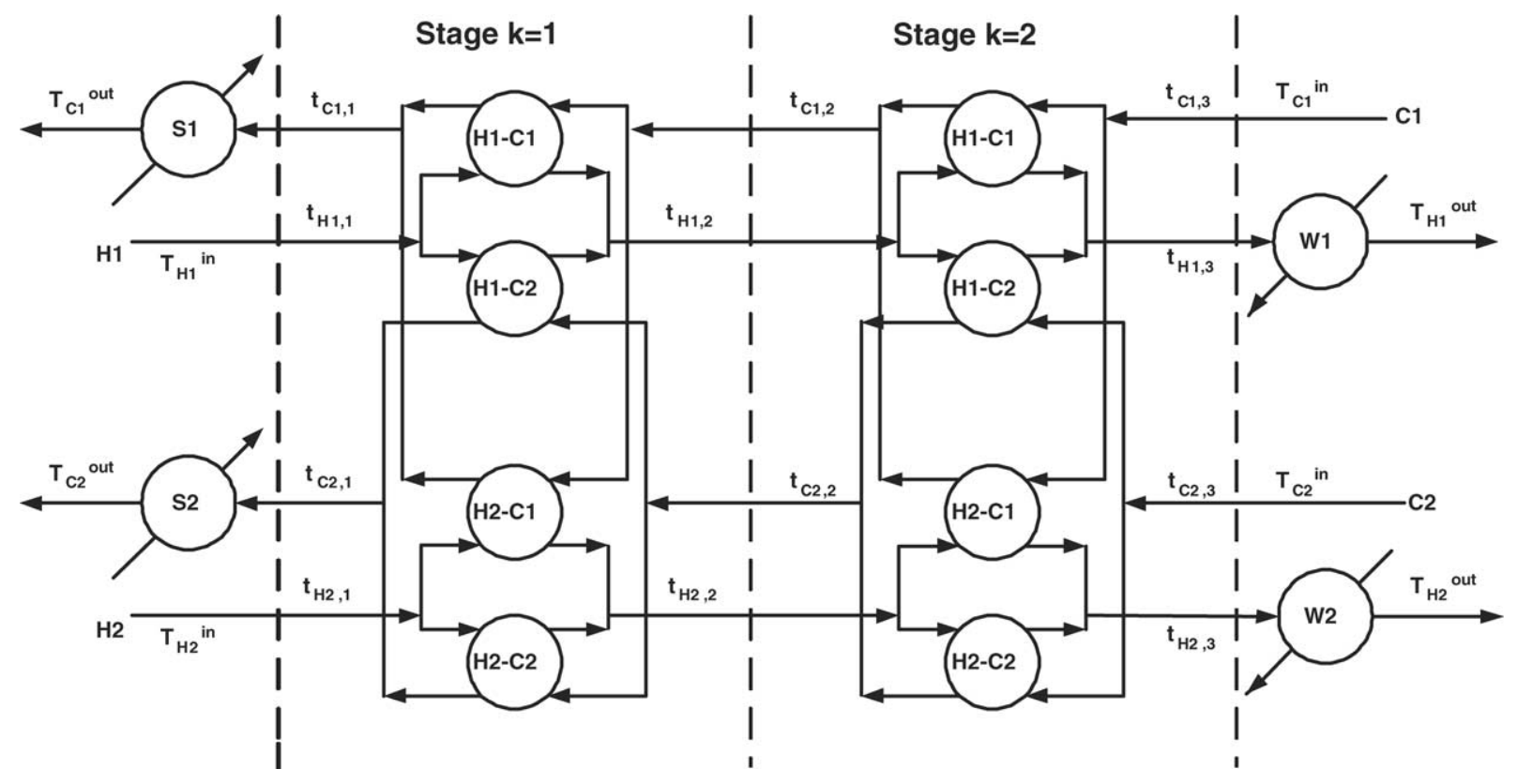

Fig. 1. The two-stage superstructure. 


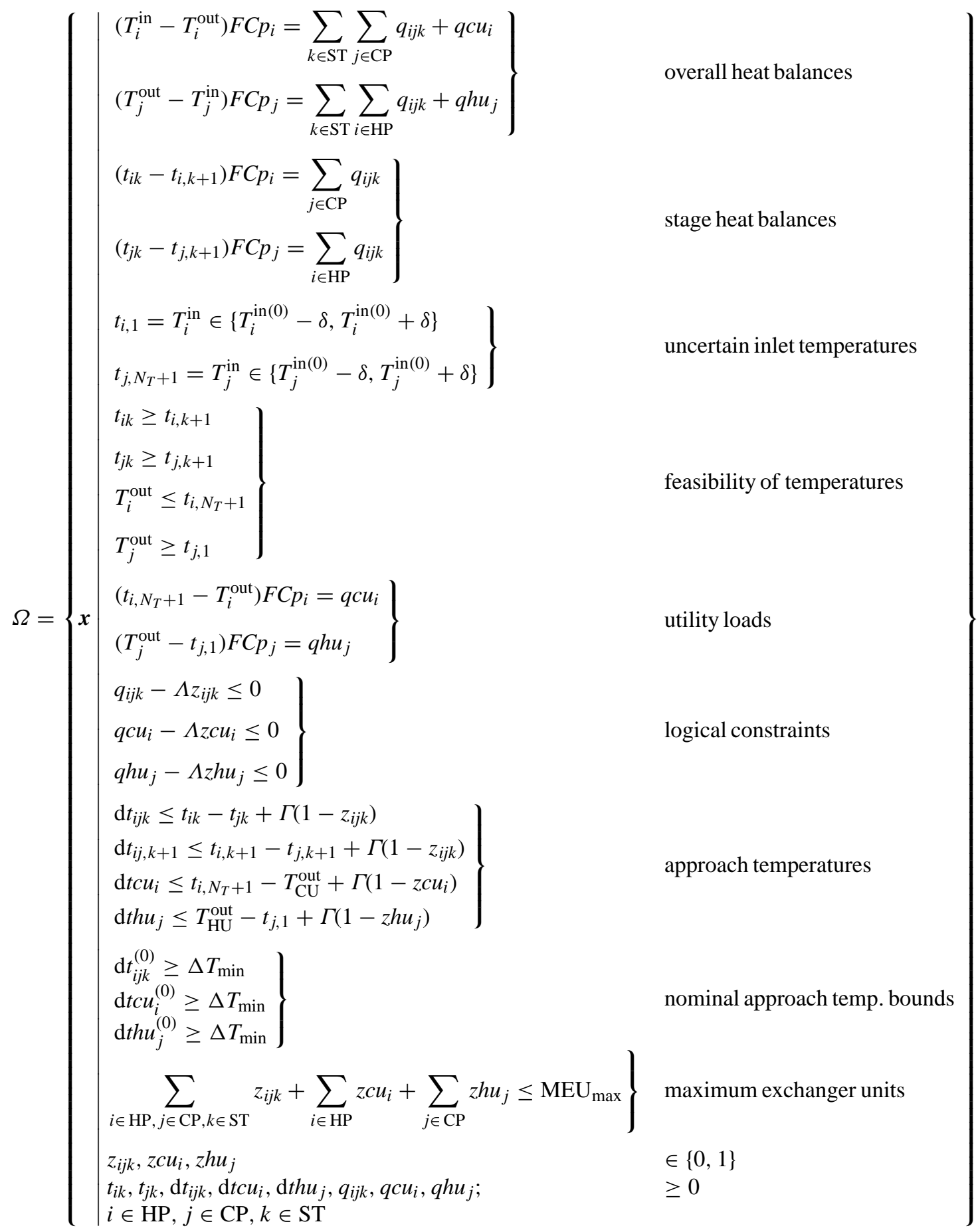

where $\boldsymbol{x}$ and $\Omega$ denote variables for design and the feasible searching space, respectively; $\delta$ is the targeted flexibility for source-stream temperatures [10]; the superscript ${ }^{(0)}$ denotes the nominal condition; and the upper bound for unit numbers is $\mathrm{MEU}_{\max }$. The searching space is comprised of all heat balances constraints and relevant logical constraints. As pointed out in [6-10], this problem is difficult to solve directly since it involves a max-min-max constraint that leads to a non-differentiable global optimization problem. For the HEN synthesis problem with uncertain source-stream tem- peratures, the feasible region defined by the reduced inequality constraints is convex [6], so the critical point that limits the solution lies at a vertex of the polyhedral region of uncertainty. For the problem of Eq. (7) with $N\left(\leq N_{\mathrm{H}}+N_{\mathrm{C}}\right)$ uncertain source-stream temperatures, the vertex-based formulation is given as follows [10]:

$\min _{\boldsymbol{x}_{\mathrm{V}} \in \Omega_{\mathrm{V}}} J^{(0)}=\sum_{i \in \mathrm{HP}} q c u_{i}^{(0)}+\sum_{j \in \mathrm{CP}} q h u_{j}^{(0)}$ 
or

$$
\begin{aligned}
& \min _{\boldsymbol{x}_{\mathrm{V} \in \Omega_{\mathrm{V}}} J^{(\mathrm{ave})}}=\frac{1}{N_{\mathrm{V}}+1} \sum_{n \in\{0\} \cup \mathrm{VT}}\left(\sum_{i \in \mathrm{HP}} q c u_{i}^{(n)}+\sum_{j \in \mathrm{CP}} q h u_{j}^{(n)}\right) \\
& \boldsymbol{x}_{\mathrm{V}}=\left\{\begin{array}{c}
z_{i j k}, z c u_{i}, z h u_{j} ; \mathrm{d} t_{i j k}^{(n)}, \mathrm{d} t c u_{i}^{(n)}, \mathrm{d} t h u_{j}^{(n)} ; \\
t_{i k}^{(n)}, t_{j k}^{(n)} ; q_{i j k}^{(n)}, q c u_{i}^{(n)}, q h u_{j}^{(n)} ; \\
i \in \mathrm{HP}, j \in \mathrm{CP}, k \in \mathrm{ST}, n \in\{0\} \cup \mathrm{VT}
\end{array}\right\}
\end{aligned}
$$

Here, $r_{i}^{(n)}$ and $r_{j}^{(n)}$ are the vertex identifiers which take values of $N_{\mathrm{V}}=2^{\left(N_{\mathrm{H}}+N_{\mathrm{C}}\right)}$ combinations of +1 and -1 , see Table 1 in [10]. Therein, Eq. (4) considers nominal utilities and Eq. (5) takes into account the average of all vertical operating points.

For the benchmark example mentioned in [10], a 2-hot/2-cold streams problem along with heating steam and cooling water, the maximal allowable variation of various possible minimum-utility HEN structures is examined by

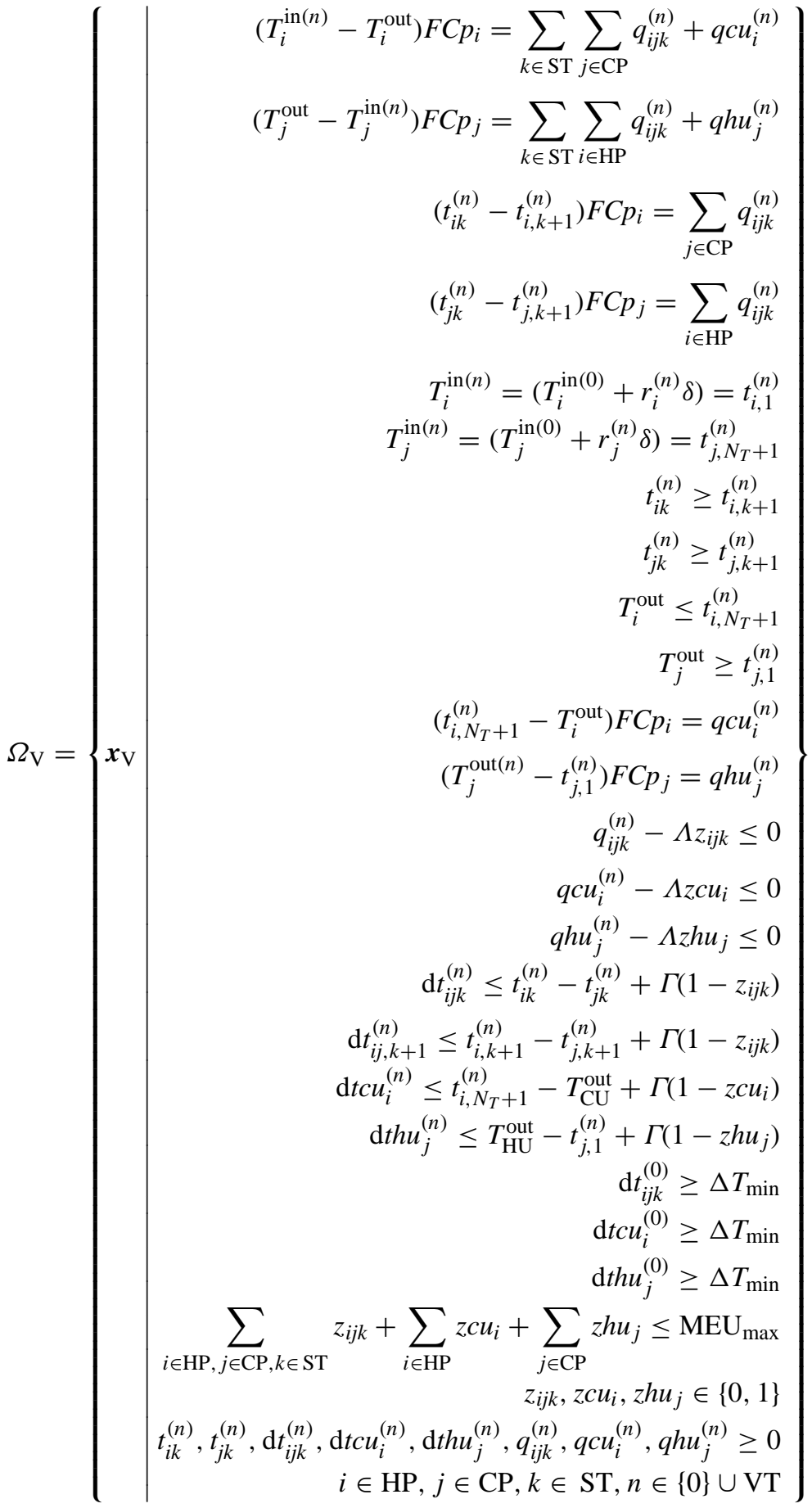


Table 1

Problem data of example 1

\begin{tabular}{llll}
\hline Process streams and utilities & Heat-capacity flow rate FCp $(\mathrm{kW} / \mathrm{K})$ & Input temperature $T^{\text {in }}(\mathrm{K})$ & Output temperature $T^{\text {out }}(\mathrm{K})$ \\
\hline Hot stream 1 (H1) & 10 & 650 & 370 \\
Hot stream 2 (H2) & 20 & 590 & 370 \\
Cold stream 1 (C1) & 15 & 410 & 650 \\
Cold stream 2 (C) & 13 & 350 & 500 \\
Hot utility (HU) & - & 680 & 680 \\
Cold utility (CU) & - & 300 & 320
\end{tabular}

$\Delta T_{\min }=10 \mathrm{~K}$.

Table 2

The resulting HEN structure of example 1 with increasing flexibility target $(\epsilon \rightarrow 0$, see Table 2 of [10] for labeling HEN structures)

\begin{tabular}{llc}
\hline Specified $\delta$ value & Resulting HEN structure & Maximum flexibility $\delta^{*}$ \\
\hline $0 \sim 36.95$ & A & 36.95 \\
$36.95+\epsilon$ & B1 & 70.63 \\
40 & B7 $^{\mathrm{a}}$ & 90.00 \\
50 & $\mathrm{~B} 6$ & 90.00 \\
60 & $\mathrm{~B}^{\mathrm{a}}$ & 70.63 \\
70 & $\mathrm{~B}^{\mathrm{a}}$ & 90.00 \\
80 & $\mathrm{~B} 7^{\mathrm{a}}$ & 90.00 \\
90 & $\mathrm{~B} 7^{\mathrm{a}}$ & 90.00 \\
$90+\epsilon$ & $\mathrm{C} 2$ & 120.0 \\
$120-150$ & $\mathrm{D}$ & 150.0 \\
\hline
\end{tabular}

increasing the $\delta$ value over $\delta \in[0,150]$ and solving Eq. (4) or Eq. (5). There are 18 sets of structure-determining binary variables and 11 of them possess unique structures (see Table 2 of [10]). However, we found that the resulting HEN structures with increasing flexibility targets are prone to variation, and the true maximal flexibilities of these HEN structures are usually greater than the required targets, as illustrated in Table 2. Similar results can also be found should restricted heat-load constraints on vertices, as shown in Eqs. (8) and (9), be taken into consideration in the HEN synthesis.

$\min _{\boldsymbol{x}_{\mathrm{V}} \in \Omega \mathrm{V} \cap \Omega_{\mathrm{load}}} J^{(0)}=\sum_{i \in \mathrm{HP}} q c u_{i}^{(0)}+\sum_{j \in \mathrm{CP}} q h u_{j}^{(0)}$

or

$$
\begin{aligned}
& \min _{\boldsymbol{x}_{\mathrm{V} \in \Omega_{\mathrm{V}} \cap \Omega_{\text {load }}} J^{(\mathrm{ave})}=} \frac{1}{N_{\mathrm{V}}+1} \times \sum_{n \in\{0\} \cup \mathrm{VT}} \\
& \times\left(\sum_{i \in \mathrm{HP}} q c u_{i}^{(n)}+\sum_{j \in \mathrm{CP}} q h u_{j}^{(n)}\right)
\end{aligned}
$$

In this paper, the targeted source-stream temperatures are directly treated as individual design objective, and the multi-criteria optimization approach is adopted for HEN synthesis. The minimizing utility and the maximizing operational flexibility can be simultaneously considered as two conflict objectives for synthesis of the network structure. Furthermore, other targets such as minimizing number of matches can also be considered, such as,

$$
\begin{aligned}
& \min _{\boldsymbol{x}_{\mathrm{V} \in \Omega_{\mathrm{V}}}} J_{1}^{(0)}=\sum_{i \in \mathrm{HP}} q c u_{i}^{(0)}+\sum_{j \in \mathrm{CP}} q h u_{j}^{(0)} \text { or } \\
& \min _{\boldsymbol{x}_{\mathrm{V} \in \Omega_{\mathrm{V}}}} J_{1}^{(\mathrm{ave})}=\frac{1}{N_{\mathrm{V}}+1} \times \sum_{n \in\{0\} \cup \mathrm{VT}} \\
& \times\left(\sum_{i \in \mathrm{HP}} q c u_{i}^{(n)}+\sum_{j \in \mathrm{CP}} q h u_{j}^{(n)}\right) \quad \text { and } \\
& \max _{\boldsymbol{x}_{\mathrm{V} \in \Omega_{\mathrm{V}}}} J_{2}=\delta \text { and } \\
& \min _{\boldsymbol{x}_{\mathrm{V} \in \Omega_{\mathrm{V}}}} J_{3}=\sum_{i \in \mathrm{HP}} \sum_{j \in \mathrm{CP}} \sum_{k \in \mathrm{ST}} z_{i j k} \\
&+\sum_{i \in \mathrm{HP}} z c u_{i}+\sum_{j \in \mathrm{CP}} z h u_{j}
\end{aligned}
$$

In such a case, a unique HEN structure with satisfactory levels in nominal or average utility consumption and operational flexibility as well as unit numbers will be obtained. A two-phase fuzzy optimization method is proposed to find a best compromised solution for the multi-criteria HEN synthesis problem, as discussed in the next section. The basic number of constraints and variables for the multi-objective MILP formulation are summarized in the following.

1. The number of constraints:

(a) for linear equality constraints: $\left(N_{\mathrm{V}}+1\right)\left[N_{T}\left(N_{\mathrm{H}}+\right.\right.$ $\left.\left.N_{\mathrm{C}}\right)+4\left(N_{\mathrm{H}}+N_{\mathrm{C}}\right)\right]$

$\Omega_{\mathrm{load}}=\left\{\begin{array}{l|l}q_{i j k}^{(n)}, q c u_{i}^{(n)}, q h u_{j}^{(n)} & \begin{array}{l}q_{i j k}^{(0)}(1-\alpha) \leq q_{i j k}^{(n)} \leq q_{i j k}^{(0)}(1+\beta) \\ q c u_{i}^{(0)}(1-\alpha) \leq q c u_{i}^{(n)} \leq q c u_{i}^{(0)}(1+\beta) \\ q h u_{j}^{(0)}(1-\alpha) \leq q h u_{j}^{(n)} \leq q h u_{j}^{(0)}(1+\beta) \\ i \in \mathrm{HP}, j \in \mathrm{CP}, k \in \mathrm{ST}, n \in \mathrm{VT}\end{array}\end{array}\right\}$ 
(b) for linear inequality constraints: $\left(N_{\mathrm{V}}+1\right)\left[\left(N_{\mathrm{H}}+\right.\right.$ $\left.\left.N_{\mathrm{C}}\right)\left(N_{T}+3\right)+3 N_{T} N_{\mathrm{H}} N_{\mathrm{C}}\right]+N_{T} N_{\mathrm{H}} N_{\mathrm{C}}+N_{\mathrm{H}}+N_{\mathrm{C}}$.

2. The number of variables:

(a) binary variables: $N_{\mathrm{H}} N_{\mathrm{C}} N_{T}+N_{\mathrm{H}}+N_{\mathrm{C}}$;

(b) positive continuous variables: $\left(N_{\mathrm{V}}+1\right)\left[N_{T}\left(2 N_{\mathrm{H}} N_{\mathrm{C}}+\right.\right.$ $\left.\left.N_{\mathrm{H}}+N_{\mathrm{C}}\right)+2\left(N_{\mathrm{H}}+N_{\mathrm{C}}\right)\right]+1$.

\section{Fuzzy multi-criteria optimization}

Consider the multi-criteria optimization problem defined in Eq. (11). Because of the fact that these objective functions usually conflict with each other in practice, the optimization of one objective implies the sacrifice of other targets; it is thus impossible to attain their own optima, $J_{S}$, $s \in \mathcal{S}=[1, \ldots, S]$, simultaneously. Therefore, the decision maker (DM) must make some compromise among these goals. In contrast to the optimality used in single objective optimization problems, Pareto optimality characterizes the solutions in a multi-objective optimization problem [13].

The weighting-sum method, among methods found in literatures for solving multi-objective optimization problems, is the one that is used most often. Basing on the subjective comprehension for each objective, the DM of this method can weigh and sum up these objectives into a scalar form, and then find the solution by any existing single-objective optimization method. However, as the situation of combining weighting factors becomes more complex, this method becomes more tedious and the solution could be still invalid. Moreover, it is difficult for the DM to attribute a set of incompatible objectives, such as utility consumption, operational flexibility, or number of matches in a heat exchanger network, without knowledge of the possible level of attainment for these objectives. The physical meaning of the final scalar objective function is thus usually vague.

In this work, we adopt the fuzzy set theory [14] to deal with the multi-objective optimization problem. By considering the uncertain property of human thinking, it is quite natural to assume that the DM has a fuzzy goal, $\mathcal{J}_{s}$, to describe the objective $J_{s}$ with an interval $\left[J_{s}^{1}, J_{s}^{0}\right]$. For the sth objective to be minimized, it is quite satisfied as the objective value $J_{s} \leq J_{s}^{1}$, and is unacceptable as $J_{s} \geq J_{s}^{0}$. The degree of satisfaction decreases as the objective value increases from $J_{s}^{1}$ to $J_{s}^{0}$. A strictly monotonic decreasing membership function, $\mu_{\mathcal{J}_{s}}\left(J_{s}\left(\boldsymbol{x}_{\mathrm{V}}\right)\right) \in[0,1]$, can be used to characterize such a transition from the objective value to the degree of satisfaction, therein value of 1 denotes absolutely satisfactory, and 0 means unacceptable. Notably, an interval of $\left[J_{s}^{0}, J_{s}^{1}\right]$ and a monotonic increasing membership function should be used for defining a fuzzy objective to be maximized. Without loss of generality, we will adopt linear membership functions in the following.

$$
\begin{aligned}
& \mu_{\mathcal{J}_{s}\left(J_{s}\left(\boldsymbol{x}_{\mathrm{V}}\right)\right)} \\
& = \begin{cases}1 ; & \text { for } \quad J_{s}^{1} \geq J_{s} \\
\frac{J_{s}^{0}-J_{s}}{J_{s}^{0}-J_{s}^{1}} & \text { for } \quad J_{s}^{1} \leq J_{s} \leq J_{s}^{0} \quad \text { for } J_{s} \text { to be minimized } \\
0 ; & \text { for } \quad J_{s} \geq J_{s}^{0}\end{cases}
\end{aligned}
$$

$$
\begin{aligned}
& \mu_{\mathcal{J}_{s}\left(J_{s}\left(\boldsymbol{x}_{\mathrm{V}}\right)\right)} \\
& = \begin{cases}1 ; & \text { for } \quad J_{s} \geq J_{s}^{1} \\
\frac{J_{s}^{1}-J_{s}}{J_{s}^{1}-J_{s}^{0}} & \text { for } \quad J_{s}^{0} \leq J_{s} \leq J_{s}^{1} \quad \text { for } J_{s} \text { to be maximized } \\
0 ; & \text { for } \quad J_{s}^{0} \geq J_{s}\end{cases}
\end{aligned}
$$

The original multi-criteria optimization problem is now converted to the one that looks for a suitable decision variable vector that can provide the maximal degree-of-satisfaction for the multiple fuzzy objectives.

$\max _{\boldsymbol{x}_{\mathrm{V}} \in \Omega_{\mathrm{V}}}\left(\mu_{\mathcal{J}_{1}}\left(\boldsymbol{x}_{\mathrm{V}}\right), \ldots, \mu_{\mathcal{J}_{S}}\left(\boldsymbol{x}_{\mathrm{V}}\right)\right)$

Under incompatible objective circumstances, a DM must make a compromise decision that provides a maximal degree-of-satisfaction for all these conflict objectives. The new optimization problem, Eq. (14), can be interpreted as the synthetic notation of a conjunction statement (maximize jointly all objectives). The result of this aggregation can be viewed as a fuzzy intersection of all fuzzy goals, $\mathcal{J}_{s}, s \in \mathcal{S}$, and is still a fuzzy set, $\mathcal{D}$.

$\mathcal{D}=\mathcal{J}_{1} \cap \ldots \cap \mathcal{J}_{S}$

The final degree-of-satisfaction resulting from certain variable set, $\mu_{\mathcal{D}}\left(\boldsymbol{x}_{\mathrm{V}}\right)$ can be determined by aggregating the degree-of-satisfaction for all objectives, $\mu_{\mathcal{J}_{s}}\left(\boldsymbol{x}_{\mathrm{V}}\right), s \in \mathcal{S}$, via specific fuzzy intersection operator, $\mathbb{T}$.

$\mu_{\mathcal{D}}\left(\boldsymbol{x}_{\mathrm{V}}\right)=\mathbb{T}\left(\mu_{\mathcal{J}_{1}}\left(\boldsymbol{x}_{\mathrm{V}}\right), \ldots, \mu_{\mathcal{J}_{S}}\left(\boldsymbol{x}_{\mathrm{V}}\right)\right)$

The fundamental properties for a fuzzy set and the related operators can be found in [15]. As the firing level for each policy is determined by the above procedure, the best solution, $\boldsymbol{x}_{\mathrm{V}}^{*}$, with the maximal firing level, $\mu_{\mathcal{D}}\left(x_{\mathrm{V}}^{*}\right)$, can be selected.

$\max _{\boldsymbol{x}_{\mathrm{V}} \in \Omega_{\mathrm{V}}} \mu_{\mathcal{D}}\left(\boldsymbol{x}_{\mathrm{V}}\right)=\max _{\boldsymbol{x}_{\mathrm{V}} \in \Omega_{\mathrm{V}}} \mathbb{T}\left(\mu_{\mathcal{J}_{1}}\left(\boldsymbol{x}_{\mathrm{V}}\right), \ldots, \mu_{\mathcal{J}_{S}}\left(\boldsymbol{x}_{\mathrm{V}}\right)\right)$

Using the fuzzy intersection operator, the original multiobjective optimization problem, Eq. (11), is converted into a single objective problem, Eq. (17). Several operators for implementing fuzzy intersection can be selected for $\mathbb{T}$, therein two most popular ones are shown below.

$\mathbb{T}\left(\mu_{\mathcal{J}_{1}}, \ldots, \mu_{\mathcal{J}_{S}}\right)$
$= \begin{cases}\min \left(\mu_{\mathcal{J}_{1}}, \ldots, \mu_{\mathcal{J}_{S}}\right) & \mathbb{T}=\text { minimum } \\ \frac{\left(\mu_{\mathcal{J}_{1}}+\ldots+\mu_{\mathcal{J}_{S}}\right)}{S} & \mathbb{T}=\text { average }\end{cases}$

The average operator simultaneously takes all membership values into account. But, no guarantee can be made for any 
single objective. The minimum operator concentrates on improving the worst scenario. However, the minimum operator may result in multiple solutions since contribution of those objectives with membership values greater than the minimum one would not be cared. We thus combine these two fuzzy intersection operators to take advantages of both and propose a two-phase optimization procedure $[11,12]$. The minimum operator is first used in phase I to find the least degree of satisfaction for the worst objective, then the average operator is applied in phase II to promote satisfaction levels of all objectives with guaranteed least membership value. The merit of this method is that we can not only obtain the unique optimal solution by using average operator but also guarantee each objective to go after their own maximum on the basis of taking the least degree of satisfaction as the lower-bound constraint. So, now we can summarize the procedure of the two-phase fuzzy satisfying approach for the multi-criteria optimization problem.

Step 1. For a function to be minimized, determine its ideal solution and anti-ideal solutions by directly minimizing and maximizing the objective function.

$$
\min _{\boldsymbol{x}_{\mathrm{V}} \in \Omega_{\mathrm{V}}} J_{s}=\underline{J}_{s}^{1}
$$

(ideal solution of $J_{S}$, totally acceptable

$$
\begin{aligned}
& \max _{\boldsymbol{x}_{\mathrm{V}} \in \Omega_{\mathrm{V}}} J_{s}=\bar{J}_{s}^{0} \\
& \quad \text { (anti }- \text { ideal solution of } J_{s}, \\
& \quad \text { unacceptable value) }
\end{aligned}
$$

Notably, direct maximization and minimization should be taken for a maximizing objective to obtain the ideal and anti-ideal solutions.

Step 2. Based on the importance of different objective functions and the acceptable ranges for objective values, subjectively select suitable lower/upper bounds, $\underline{J}_{s}^{1} \leq J_{s}^{1} \leq J_{s}^{0} \leq \bar{J}_{s}^{0}$ for minimizing objective and $\underline{J}_{s}^{0} \leq J_{s}^{0} \leq J_{s}^{1} \leq \bar{J}_{s}^{1}$ for maximizing objective. Define membership functions for multiple fuzzy objectives as given in Eqs. (12) and (13).

Step 3. (Phase I). Use the minimum operator to find the maximal degree of satisfaction for the worst objective, $\mu^{\min }$.

$$
\begin{aligned}
& \max _{\boldsymbol{x}_{\mathrm{V}} \in \Omega_{\mathrm{V}}} \mu_{\mathcal{D}}=\max _{\boldsymbol{x}_{\mathrm{V}} \in \Omega_{\mathrm{V}}} \\
& \min \left(\mu_{\mathcal{J}_{1}}, \ldots, \mu_{\mathcal{J}_{S}}\right)=\mu^{\min }
\end{aligned}
$$

Step 4. (Phase II). Use the average operator to simultaneously promote satisfaction levels of all objectives with new constraints of guaranteed minimal degree of satisfaction.

$\max _{\boldsymbol{x}_{\mathrm{V}} \in \Omega_{\mathrm{V}}^{+}} \mu_{\mathcal{D}}=\max _{\boldsymbol{x}_{\mathrm{V}} \in \Omega_{\mathrm{V}}^{+}} \frac{\mu_{\mathcal{J}_{1}}+\cdots+\mu_{\mathcal{J}_{S}}}{S}$

where

$\Omega_{\mathrm{V}}^{+}=\Omega_{\mathrm{V}} \cap\left\{\mu_{\mathcal{J}_{s}} \geq \mu^{\min }, \forall s \in \mathcal{S}\right\}$
The new feasible space guarantees the least degree of satisfaction for each fuzzy objective.

\section{Numerical example}

Two numerical examples adapted from $[4,10]$ are supplied to demonstrate the efficacy of proposed HEN synthesis strategy. To solve this MO-MILP for the HEN model, GAMS [16] and CPLEX are used as the modeling environment and the MIP solver, respectively. The computing machine is a personal computer with an Intel Pentium IV 2.26 GHz CPU.

Example 1. The 2-hot/2-cold streams example studied by [10], with problem data presented in Table 1, is illustrated. With these parameters, the multi-objective MILP formulation has 408 linear equality constraints, 760 linear inequality constraints, 12 binary variables, and 545 positive continuous variables. Notably, the restriction of $\mathrm{MEU}_{\max }=6$ in Eq. (7) will be removed should the minimum number of matches be simultaneously taken into account as one of the design objectives.

According to the model formulation and the problem data, we solve the multi-criteria MILP synthesis problem by using the fuzzy optimization procedure discussed in Section 3. The ideal and anti-ideal solutions, obtained by sequentially minimizing and maximizing each objective function, are shown in Table 3. Within these lower/upper limiting values, several preference intervals are subjectively selected to establish linear membership functions for the fuzzy objectives. In implementing the two-phase fuzzy optimization, we firstly use the minimum operator to maximize the degree of satisfaction for the worst target, $\mu^{\mathrm{min}}$. The average operator is then applied to optimize the aggregated objectives with guaranteed level of satisfaction.

At first, only two conflict objectives are considered: the minimal utility consumption and the maximal flexibility to all source-stream temperatures. And then the third objective, the minimal number of matches, would be appended. Results of two-phase fuzzy optimization with preference intervals of $[2550,12750]$ or $[2550,8850]$ for utility, [0, 150], [40, 90] or [40, 70] for flexibility, and [4, 7] for unit numbers, along with either or not considering restrictions on heat loads at extreme operating points, are listed in Table 4. The resulting HEN structures are also depicted in Fig. 2. Notably, the reduced range of flexibility, [40, 90], implies that the required minimum tolerance for temperature deviation is at least $40 \mathrm{~K}$ and a tolerance of maximum temperature deviation for $90 \mathrm{~K}$

Table 3

The ideal/anti-ideal solutions of various objectives of example 1

\begin{tabular}{lrr}
\hline Objective function & Ideal solution $J_{i}^{1}$ & Anti-ideal solution $\bar{J}_{i}^{0}$ \\
\hline$J_{1}^{(0)}$ utility $(\mathrm{kW})$ & 2550 & 12750 \\
$J_{2}$ flexibility $(K)$ & 150 & 0 \\
$J_{3}$ units & 4 & 12 \\
\hline
\end{tabular}


Table 4

Results of HEN synthesis for example 1 using two-phase optimization when simultaneously considering minimal utility and maximal flexibility (cases I-IV), and additional objective of minimal units (cases V and VI) with different preference intervals, and with or without considering restriction on heat loads at vertices $(\alpha=\beta=0.6)$

\begin{tabular}{|c|c|c|c|c|c|c|c|c|c|c|c|}
\hline \multirow[t]{2}{*}{ Case } & \multirow[t]{2}{*}{ Preference intervals } & \multirow[t]{2}{*}{ Heat load } & \multirow[t]{2}{*}{ Phase } & \multicolumn{4}{|c|}{ Minimum utility } & \multicolumn{2}{|c|}{ Maximum flexibility } & \multicolumn{2}{|c|}{ Minimum units } \\
\hline & & & & Hot & Cold & Total & Satisfaction & Flexibility & Satisfaction & Units & Satisfaction \\
\hline I & {$[2550,12750]$} & No & I & 1300 & 2950 & 4250 & 0.833 & 120.0 & 0.800 & 6 & - \\
\hline$(\mathrm{C} 2)$ & {$[0,150],[-, 6]$} & & II & 1300 & 2950 & 4250 & 0.833 & 120.0 & 0.800 & 6 & - \\
\hline II & {$[2550,8850]$} & No & I & 1050 & 2700 & 3750 & 0.810 & 80.5 & 0.810 & 6 & - \\
\hline (B7) & {$[40,90],[-, 6]$} & & II & 1050 & 2700 & 3750 & 0.810 & 90.0 & 1.00 & 6 & - \\
\hline \multirow[t]{2}{*}{ III } & {$[2550,8850]$} & Yes & I & 2190 & 3840 & 6030 & 0.448 & 62.4 & 0.448 & 6 & - \\
\hline & {$[40,90],[-, 6]$} & & II & 2190 & 3840 & 6030 & 0.448 & 62.4 & 0.448 & 6 & - \\
\hline \multirow[t]{2}{*}{ IV } & {$[2550,8850]$} & Yes & $\mathrm{I}$ & 1893 & 3543 & 5436 & 0.542 & 56.3 & 0.542 & 6 & - \\
\hline & {$[40,70],[-, 6]$} & & II & 1893 & 3543 & 5436 & 0.542 & 56.3 & 0.542 & 6 & - \\
\hline \multirow[t]{2}{*}{$\mathrm{V}$} & {$[2550,8850]$} & No & I & 1300 & 2950 & 4250 & 0.730 & 73.3 & 0.667 & 5 & 0.667 \\
\hline & {$[40,90],[4,7]$} & & II & 1300 & 2950 & 4250 & 0.730 & 74.2 & 0.685 & 5 & 0.667 \\
\hline \multirow[t]{2}{*}{ VI } & {$[2550,8850]$} & Yes & I & 2550 & 4200 & 6750 & 0.333 & 56.7 & 0.333 & 6 & 0.333 \\
\hline & {$[40,90],[4,7]$} & & II & 2550 & 4200 & 6750 & 0.333 & 69.8 & 0.597 & 6 & 0.333 \\
\hline
\end{tabular}

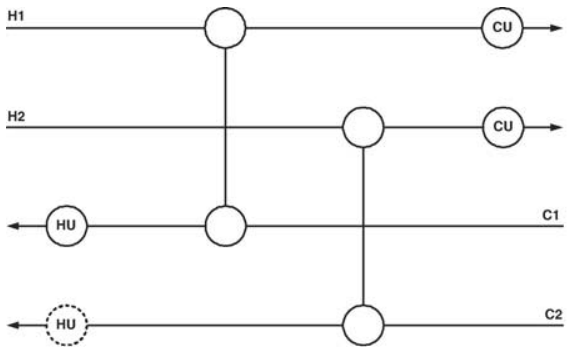

Case I

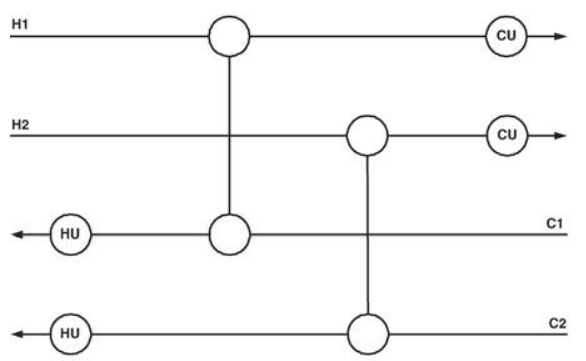

Case III

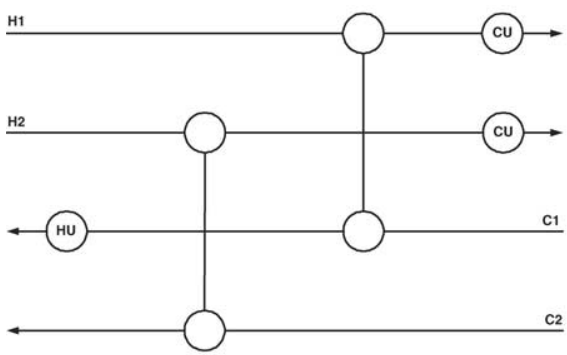

Case V

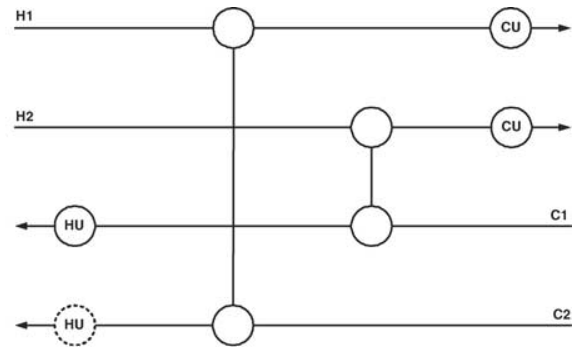

Case II

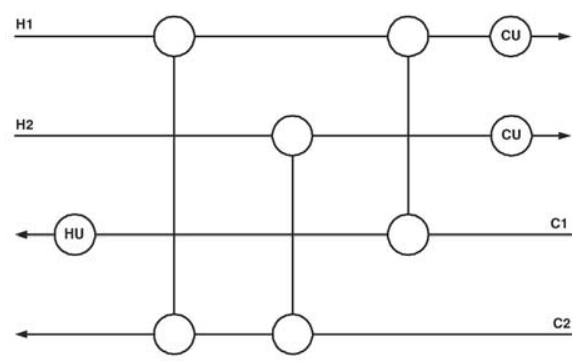

Case IV

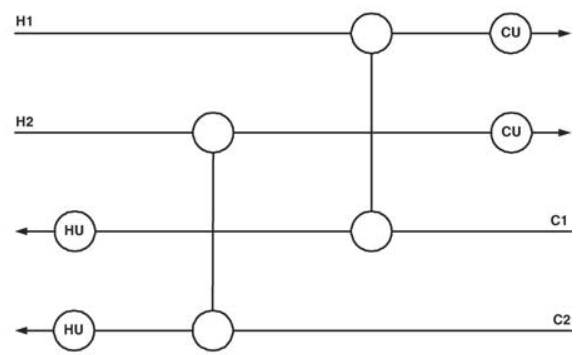

Case VI

Fig. 2. The HEN structures for cases I-VI of example 1. 
Table 5

Problem data of example 2

\begin{tabular}{lcll}
\hline $\begin{array}{l}\text { Process streams } \\
\text { and utilities }\end{array}$ & $\begin{array}{l}\text { Heat-capacity } \\
\text { flow rate FCp } \\
(\mathrm{kW} / \mathrm{K})\end{array}$ & $\begin{array}{l}\text { Input } \\
\text { temperature } \\
T^{\text {in }}(\mathrm{K})\end{array}$ & $\begin{array}{l}\text { Output } \\
\text { temperature } \\
T^{\text {out }}(\mathrm{K})\end{array}$ \\
\hline Hot stream 1 (H1) & 6 & 500 & 320 \\
Hot stream 2 (H2) & 4 & 480 & 380 \\
Hot stream 3 (H3) & 6 & 460 & 360 \\
Hot stream 4 (H4) & 20 & 380 & 360 \\
Hot stream 5 (H5) & 12 & 380 & 320 \\
Cold stream 1 (C1) & 18 & 290 & 660 \\
Hot utility (HU) & - & 700 & 700 \\
Cold utility (CU) & - & 300 & 320
\end{tabular}

$\Delta T_{\min }=10 \mathrm{~K}$.

Table 6

The ideal/anti-ideal solutions of various objectives of example 2

\begin{tabular}{lrc}
\hline Objective function & Ideal solution $\underline{J}_{i}^{1}$ & Anti-ideal solution $\bar{J}_{i}^{0}$ \\
\hline$J_{1}^{(0)}$ utility $(\mathrm{kW})$ & 3780 & 9860 \\
$J_{2}$ flexibility $(\mathrm{K})$ & 20 & 0 \\
$J_{3}$ units & 6 & 31 \\
\hline
\end{tabular}

is absolutely satisfied. We choose 90 as the maximum deviation for most cases since it is the maximal tolerance for the HEN with heat load restrictions on vertices.

As shown in Table 4 and Fig. 2, the HEN structure in case I is the same as $C 2$ of [10], therein the maximum flexibility to temperatures is explicitly given as 120 . In case II, the $B 7$ structure of [10] can be obtained when the tolerable upper bound for utility consumption is decreased from 12750 to 8850 , and the preference interval for flexibility is reduced from $[0,150]$ to $[40,90]$. The resulting utility consumption will be reduced from 4250 (Case I) to 3750 due to the restriction of preference intervals, and the flexibility, 90 , is equivalent to the maximum of the $B 7$ structure.

All other conditions are equal to those in case II, case III includes heat-load restrictions $(\alpha=\beta=0.6)$ on various vertical operating points as additional constraints. The prices of such additional restrictions are increased utility consumption from 3750 to 6030 and decreased flexibility to temperature deviation from 90 to 62.4 , which is still significantly greater than the minimum targeted value, 40 . In case $\mathrm{IV}$, the preference interval for temperature deviation is further reduced to $[40,70]$. It is found that the resulting HEN has smaller utility consumption, 5438, with the expense of further reduction on flexibility, 56.3, since our desideratum for flexibility has been made lower.

Cases V and VI take into account the unit number as the third design objective, where heat-load restrictions on vertices are either included or not. In case V, it is found that the unit number is only five, flexibility to temperature deviation is 74.2 , and the utility consumption is 4250 , a little more than $B 4$ and $B 5$ of [10]. In case VI where heat-load restrictions on vertices are further included, the total unit number becomes six, the total utility is increased to 6750 with a reduced maximum temperature flexibility of 69.8 .

From these results, it is found that the proposed multi-criteria synthesis strategy can attain a definite and compromised solution for a problem with assorted conflict objectives. The preference intervals of various objectives have significant effects on final HEN structures. Such acceptable and/or preference intervals can also reflect the importance of different objective functions. Should one specific objective is emphasized, a tighter restriction or shrinking span should be placed on its acceptable ranges.

Table 7

Results of HEN synthesis for example 2 using two-phase optimization when simultaneously considering minimal utility, maximal flexibility and minimal units with different preference intervals, and with or without considering restriction on heat loads $(\alpha=\beta=0.6)$

\begin{tabular}{|c|c|c|c|c|c|c|c|c|c|c|c|}
\hline \multirow[t]{2}{*}{ Case } & \multirow[t]{2}{*}{ Preference intervals } & \multirow[t]{2}{*}{ Heat load } & \multirow[t]{2}{*}{ Phase } & \multicolumn{4}{|c|}{ Minimum utility } & \multicolumn{2}{|c|}{ Maximum flexibility } & \multicolumn{2}{|c|}{ Minimum units } \\
\hline & & & & Hot & Cold & Total & Satisfaction & Flexibility & Satisfaction & Units & Satisfaction \\
\hline \multirow[t]{2}{*}{ I } & {$[3780,9860]$} & \multirow[t]{2}{*}{ No } & I & 3863 & 403 & 4266 & 0.920 & 18.4 & 0.920 & 8 & 0.920 \\
\hline & {$[0,20],[6,31]$} & & II & 3793 & 333 & 4126 & 0.943 & 20.0 & 1.00 & 8 & 0.920 \\
\hline \multirow[t]{2}{*}{ II } & {$[3780,4930]$} & \multirow[t]{2}{*}{ No } & I & 3712 & 252 & 3964 & 0.840 & 16.8 & 0.840 & 10 & 0.840 \\
\hline & {$[0,20],[6,31]$} & & II & 3660 & 200 & 3860 & 0.930 & 20.0 & 1.00 & 10 & 0.840 \\
\hline \multirow[t]{2}{*}{ III } & {$[3780,4930]$} & \multirow[t]{2}{*}{ No } & I & 3811 & 351 & 4162 & 0.667 & 13.3 & 0.667 & 8 & 0.667 \\
\hline & {$[0,20],[6,12]$} & & II & 3793 & 333 & 4126 & 0.699 & 20.0 & 1.00 & 8 & 0.667 \\
\hline \multirow[t]{2}{*}{ IV } & {$[3780,4930]$} & \multirow[t]{2}{*}{ No } & I & 3811 & 351 & 4162 & 0.667 & 16.7 & 0.667 & 8 & 0.667 \\
\hline & {$[10,20],[6,12]$} & & II & 3793 & 333 & 4126 & 0.699 & 20.0 & 1.00 & 8 & 0.667 \\
\hline \multirow[t]{2}{*}{ V } & {$[3780,9860]$} & \multirow[t]{2}{*}{ Yes } & I & 3980 & 520 & 4500 & 0.882 & 10.6 & 0.882 & 8 & 0.920 \\
\hline & {$[0,12],[6,31]$} & & II & 3980 & 520 & 4500 & 0.882 & 12.0 & 1.00 & 8 & 0.920 \\
\hline \multirow[t]{2}{*}{ VI } & {$[3780,4930]$} & \multirow[t]{2}{*}{ Yes } & I & 3742 & 282 & 4024 & 0.787 & 9.4 & 0.787 & 11 & 0.800 \\
\hline & {$[0,12],[6,31]$} & & II & 3742 & 282 & 4024 & 0.787 & 9.4 & 0.787 & 11 & 0.800 \\
\hline \multirow[t]{2}{*}{ VII } & {$[3780,4930]$} & \multirow[t]{2}{*}{ Yes } & I & 3860 & 400 & 4260 & 0.583 & 6.99 & 0.583 & 8 & 0.667 \\
\hline & {$[0,12],[6,12]$} & & II & 3860 & 400 & 4260 & 0.583 & 10.0 & 0.833 & 8 & 0.667 \\
\hline \multirow[t]{2}{*}{ VIII } & {$[3780,4930]$} & \multirow[t]{2}{*}{ Yes } & I & 3860 & 400 & 4260 & 0.583 & 9.5 & 0.583 & 8 & 0.667 \\
\hline & {$[6,12],[6,12]$} & & II & 3860 & 400 & 4260 & 0.583 & 10.0 & 0.667 & 8 & 0.667 \\
\hline
\end{tabular}




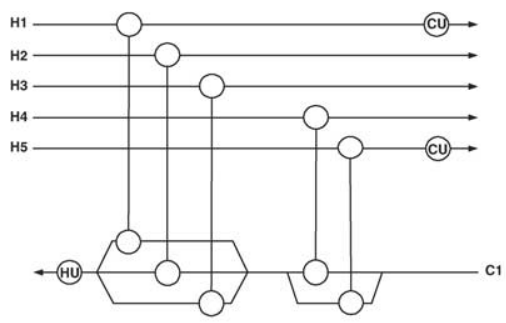

Case I

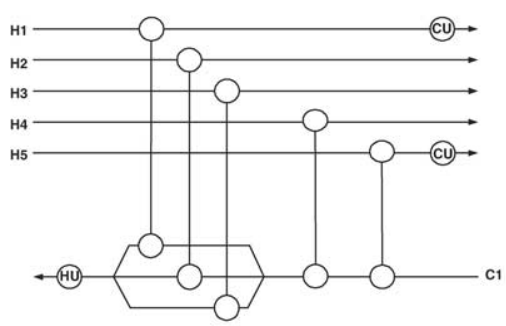

Case III

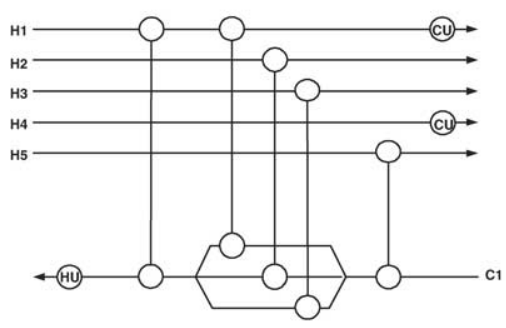

Case V

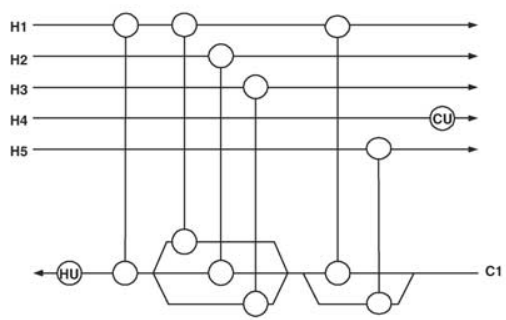

Case VII

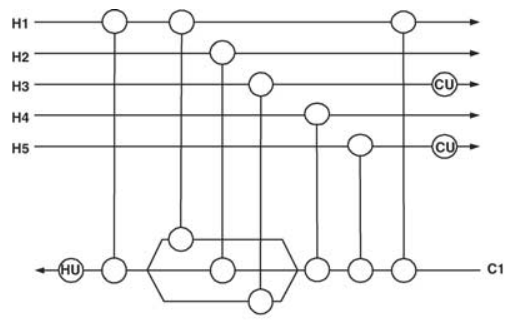

Case II

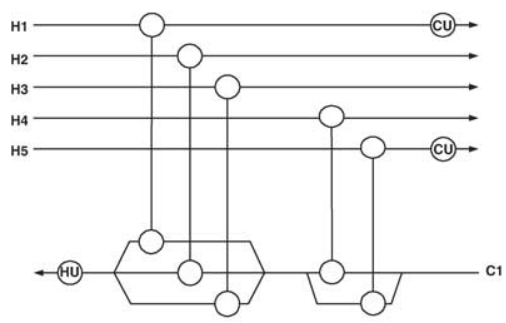

Case IV

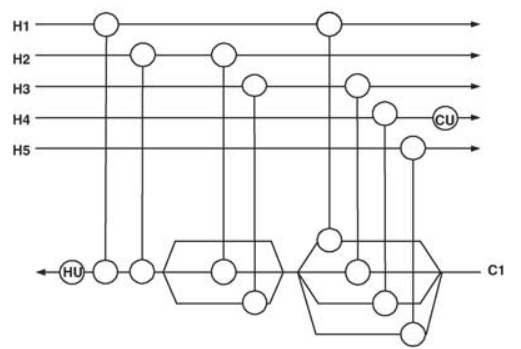

Case VI

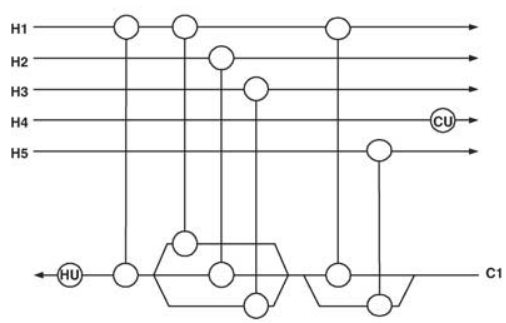

Case VIII

Fig. 3. The HEN structures for cases I-VIII of example 2.

Observing the results shown in Table 4, we also discover that the integrated two-phase method can bring the merits of the minimum and average operators together. The minimum operator maximize the degree of satisfaction for the worst objective, and can result in a harmonious solution with satisfaction levels for objective functions that are equal or close to each other. The average operator, on the other hand, can sometimes promote satisfaction levels for other objectives with guaranteed minimum value, such as Cases II, V, and VI. Owing to these advantages, the two-phase method can thus provide the best compromised HEN configuration.

Example 2. This problem consists of five hot streams and one cold stream, $\left(N_{\mathrm{H}}=5, N_{\mathrm{C}}=1\right)$, along with steam and cooling water as utilities [3]. The problem data are listed in Table 5. The number of superstructure stages is set as $N_{T}=5$. With these parameters, the MO-MILP formulation has 3510 linear equality constraints, 8026 linear inequality constraints, 31 binary variables, and 5981 positive continuous variables. The ideal and anti-ideal solutions are shown in Table 6. Various preference intervals are sequentially selected for defining the membership functions, as shown in Table 7.

We directly use the ideal/anti-ideal solutions as the preference intervals in Case I. The acceptable utility range is reduced from [3780,9860] to [3780,4930] in Case II. With such a smaller allowance interval, the resulting utility consumption will be reduced slightly from 3793 to 3660 at the expense of unit numbers increased from 8 to 10. In Case III, 
the maximum allowable unit number is changed from 31 to 12. The resulting HEN structure is slightly different to that of Case I, but the required unit numbers, utilities, and flexibility are the same as Case I. In Case IV, when the minimum flexibility requirement is upgraded from 0 to 10 , the same HEN structure of Case I results with the same levels of utility, flexibility and unit numbers. Cases V-VIII give similar results with additional heat-load restrictions $(\alpha=\beta=0.6)$ on various vertical operating points. With such additional constraints, the HEN structures use similar levels of utilities and unit numbers, but the flexibility levels are dramatically reduced. The resulting HEN structures are depicted in Fig. 3.

\section{Conclusion}

In this paper, we use the fuzzy multi-criteria optimization approach to synthesize the heat-exchanger network where some conflict design objectives such as the total utility consumption, the flexibilities to source-stream temperature variations, and even the total number of heat exchange units can be considered simultaneously. Such a flexible HEN synthesis problem can be formulated as a multi-objective mixed-integer linear programming (MO-MILP). For handling the multiple conflict design objectives, a two-phase fuzzy optimization method is proposed to attain the best compromised solution. The attractive features of the proposed MO-MILP model are that it not only considers the trade-off among the utility consumption, the source-stream temperature flexibility, and even the number of matches, but also avoids the determination of structural boundaries, as discussed in [10]. Two numerical examples with various cases are studied, demonstrating that the proposed strategy can provide a feasible compensatory solution for the multi-criteria HEN synthesis problem.

\section{Acknowledgements}

This work is supported by the National Science Council (ROC) under Contract NSC91-ET-7-002-004-ET. Partial financial support of Ministry of Economic Affairs under grant 92-EC-17-A-09-S1-019 is also acknowledged.

\section{Appendix A. Nomenclature}

$\mathrm{CP} \quad$ index set of cold process stream

$\mathrm{d} t_{i j k} \quad$ temperature approach for match $i$ and $j$ in stage $k$

$\mathrm{d} t c u_{i} \quad$ temperature approach for match $i$ and cold utility

$\mathrm{d} t h u_{j} \quad$ temperature approach for match $j$ and hot utility

$F C p \quad$ heat capacity flowrate

HP index set of hot process stream

$J \quad$ objective function

$\mathrm{MEU}_{\max }$ maximum number of heat-exchange units

$N \quad$ number of uncertain parameters

$\begin{array}{ll}N_{\mathrm{C}} & \text { number of cold streams } \\ N_{\mathrm{H}} & \text { number of hot streams } \\ N_{T} & \text { number of superstructure stages } \\ N_{\mathrm{V}} & \text { number of vertices, }=2^{N} \\ q_{i j k} & \text { heat exchanged between stream } i \text { and } j \text { in stage } k \\ q c u_{i} & \text { heat exchanged between stream } i \text { and cold utility } \\ q h u_{j} & \text { heat exchanged between stream } i \text { and hot utility } \\ r & \text { directional identifier for vertices } \\ \mathrm{ST} & \text { index set of superstructure stages } \\ S & \text { number of objectives } \\ \Delta T_{m i n} & \text { minimum approach temperature } \\ t_{i k} & \text { temperature of stream } i \text { at hot end of stage } k \\ t_{j k} & \text { temperature of stream } j \text { at hot end of stage } k \\ T & \text { temperature } \\ \mathrm{VT} & \text { index set of vertices } \\ \boldsymbol{x}, \boldsymbol{x}_{\mathrm{V}} & \text { vector of variables } \\ z_{i j k} & \text { binary variable for existence of unit for match } i \\ & \text { and } j \text { in stage } k \\ z c u_{i} & \text { binary variable for existence of unit for match } i \\ & \text { and cold utility in stage } k \\ z h u_{i} & \text { binary variable for existence of unit for match } j \\ & \text { and hot utility in stage } k\end{array}$

\section{Greek letters}

$\alpha, \beta \quad$ parameters used for restriction of heat-load deviations

$\delta \quad$ flexibility index

$\delta^{*} \quad$ flexibility index (scalar)

$\mu_{\mathcal{J}_{s}} \quad$ membership function for $\mathcal{J}_{s}$

$\mathcal{J}_{s} \quad$ a fuzzy goal

$\mathcal{S} \quad$ index set of multiple objectives

$\mathcal{D} \quad$ a fuzzy set

$\mu_{\mathcal{D}} \quad$ degree of satisfaction

$\Gamma \quad$ upper bound for temperature difference

$\Lambda \quad$ upper bound for heat exchange

$\Omega \quad$ the feasible searching region

\section{Superscripts}

$\begin{array}{ll}\text { in } & \text { inlet } \\ (n) & \text { identifier for vertices } \\ \text { out } & \text { outlet } \\ (0) & \text { identifier for nominal operating condition }\end{array}$

$\begin{array}{ll}\text { Subscripts } & \\ \text { CU } & \text { cold utility } \\ \text { HU } & \text { hot utility } \\ i & \text { index for hot process streams } \\ j & \text { index for cold process streams } \\ k & \text { index for superstructure stages } \\ s & \text { index for objectives }\end{array}$

\section{References}

[1] K.C. Furman, N.V. Sahinidis, A critical review and annotated bibliography for heat exchanger network synthesis in the 20th century, Ind. Eng. Chem. Res. 41 (2002) 2335-2370. 
[2] B. Linnhoff, E. Hindmarsh, The pinch design method for heat exchanger networks, Chem. Eng. Sci. 38 (5) (1983) 745-763.

[3] T.F. Yee, I.E. Grossmann, Z. Kravanja, Simultaneous optimization models for heat integration-I. Area and energy targeting and modeling of multi-stream exchangers, Comp. Chem. Eng. 14 (10) (1990) 11511164.

[4] T.F. Yee, I.E. Grossmann, Simultaneous optimization models for heat integration-II. Heat exchanger network synthesis, Comp. Chem. Eng. 14 (10) (1990) 1165-1184.

[5] C.A. Floudas, Nonlinear and Mixed-Integer Optimization: Fundamentals and Applications, Oxford University Press, New York, 1995.

[6] L.T. Biegler, I.E. Grossmann, A.W. Westerberg, Systematic Methods of Chemical Process Design, Prentice Hall, Englewood Cliffs, NJ, 1997.

[7] K.P. Halemane, I.E. Grossmann, Optimal process design under uncertainty, AIChE J. 29 (3) (1983) 425-433.

[8] R.E. Swaney, I.E. Grossmann, An index for operational flexibility in chemical process design, AIChE J. 31 (4) (1985) 621-630.

[9] I.E. Grossmann, C.A. Floudas, Active constraint strategy for flexibility analysis in chemical processes, Computers Chem. Eng. 11 (6) (1987) 675-693.
[10] A.E.S. Konukman, M.C. Camurdan, U. Akman, Simultaneous flexibility targeting and synthesis of minimum-utility heat exchanger networks with superstructure-based MILP formulation, Chem. Eng. Processing 41 (2002) 501-518.

[11] C.L. Chen, B.W. Wang, W.C. Lee, Multi-objective Optimization for Multi-enterprise Supply Chain Networks, Ind. Eng. Chem. Res. 42 (2003) 1879-1889.

[12] R.J. Li, E.S. Lee, Fuzzy multiple objective programming and compromise programming with Pareto optimum, Fuzzy Sets and Systems 53 (1993) 275-288.

[13] Sakawa, M., Fuzzy Sets and Interactive Multi-Objective Optimization, Plenum Press, New York, 1993.

[14] L.A. Zadeh, Fuzzy sets, Information and Control 8 (3) (1965) 338353.

[15] G.L. Klir, B. Yuan, Fuzzy Sets and Fuzzy Logics-Theory and Application, Prentice Hall, New York, 1995.

[16] A. Brooke, D. Kendrick, A. Meeraus, R. Raman, R.E. Rosenthal, GMAS: A User's Guide, GAMS Development Corporation, 1988. 